\title{
Mathematical Modeling of Dengue Disease under Random Effects
}

\author{
Zafer Bekiryazici, Mehmet Merdan, Tülay Kesemen* and Mohammed Najmuldeen \\ (Communicated by Türkan ERBAY DALKILIÇ)
}

\begin{abstract}
In this study, the deterministic mathematical model of Dengue disease is examined under Laplacian random effects. Random variables with Laplace distribution are used for randomizing the deterministic parameters. Simulations of the numerical results of the equation system are made with Monte-Carlo methods and the results are used for commenting on the disease. Comments are made on the random behavior of the components of the model after the calculation of their numerical characteristics like the expected value, variance, standard deviation, confidence interval and moments along with the coefficients of skewness and kurtosis from the results of the simulations. Results from the deterministic model are compared with the results from the random model to point out the possible contribution of random modeling to mathematical analysis studies on the disease.

Keywords: Mathematical Model; Random Effect; Simulation; Laplace Distribution.

AMS Subject Classification (2010): Primary: 34F05 ; Secondary: 82B80; 60K37; 92D30.

${ }^{*}$ Corresponding author
\end{abstract}

\section{Introduction}

A mathematical model is the expression of a system using the language of mathematics and the process of building mathematical models is called mathematical modeling. Mathematical models can be found in physics, chemistry, engineering and natural science areas along with social sciences like sociology and psychology. Events that will be modeled mathematically can be classified in two groups as deterministic and stochastic. Result can be obtained in deterministic events and this result would always be the same under the same conditions. In stochastic events, different results can be obtained under the same conditions. Differences that can occur upon use of random models for investigations of diseases should be examined by a comparison with deterministic modeling studies. Although the variations in the results of events in real life can be described in equations by the use of random variables and stochastic processes, it can be seen that mostly stochastic processes are used in mathematical modeling studies.

Dengue Fever is a tropical disease caused by the dengue virus. The disease spreads primarily by the bite of "Aedes" mosquitoes, especially of the type "Aedes aegypti". Although the disease which is spread by the bites of female Aedes aegypti mosquitoes causes certain death of mosquitoes, the mortality rate for people is very low. Muscle and joint pains, skin rashes and headaches are amongst some of the symptoms of this disease [8, 24]. The disease has become a major health problem in Southeastern Asia especially after 1960s with two of its dangerous forms DHF - Dengue Hemorrhagic Fever and DSS - Dengue Shock Syndrome. Although the disease can be seen in Americas, Africa and Mediterranean countries, it is most dangerous in West Pacific, Southeastern Asian and American regions with tropical climates [25]. The disease becomes epidemic in pre- and late-monsoon periods due to the increase in mosquito population and breeding places. While Dengue cases (DSS-DHF) were seen in

Received : 24-June-2016, Accepted : 14-October-2016 
only 9 countries before 1970, they are now seen in more than 100 countries. Latest studies show that dengue virus causes about 390 million new infections annually [2] and about 96 million of these infections occur at a critical level. Another study indicates that 3.9 billion people in 128 countries worldwide face the danger of Dengue virus. Although the first identification of Dengue virus goes back to the end of 18th century, four close types of this virus are known today. The disease caused by these viruses, which are known as DEN 1, DEN 2, DEN 3 and DEN 4, does not have a specific treatment but the mortality rate of dengue fever can be reduced to below $1 \%$ by early diagnosis, battling mosquitoes and a correct intervention [20,25]. Recovery from the disease caused by a specific type of the virus provides permanent immunity to that type but not to other types of the virus. The transmission rate of dengue virus, which can be seen in most countries controlled by the World Health Organization and threatens more than half of the world population, is rapidly increasing. Several deterministic modeling studies investigate various mathematical properties of Dengue Disease [8, 20, 24].

The randomness in the results of real life events can be described in equations by the use of random variables and stochastic processes. However, it can be seen that most of these random studies include the use of stochastic noise elements. The study of Imran M. et al. can be shown as an example of the stochastic effect terms in mathematical models [11]. Another example of stochastic models in the mathematical analysis of diseases is the study of Tan W. and $\mathrm{Wu} \mathrm{H}$. [23], where CD4+ T-cell infection by HIV is analyzed. Stochastic models are also used in other areas, as seen in the study of Pang et al., where tobacco control in China is modeled stochastically [19]. However, not many examples can be listed for random models consisting of random differential equations. The study of Merdan M. and Khaniyev T. named "On the Behaviour of Solutions under the Influence of Stochastic Effect of Avian-Human Influenza Epidemic Model" can be given as an example for these models [18], where the parameters of a deterministic model of avian-influenza disease are randomized by using normally and triangularly distributed random effects and a system of random differential equations is built. In this study, a deterministic model of dengue disease will be used to build a random model and the simulations of the numerical solutions of this system will be used to comment on the characteristics of the model components.

\section{Deterministic Model of Dengue Fever Disease}

Dengue Fever disease has been examined with deterministic models in various studies [8, 20,24]. The model given in the study of Esteva and Vargas consists of 3 nonlinear differential equations which are given as [8]:

$$
\begin{aligned}
\frac{d}{d t} S_{H}^{*} & =\mu_{H}\left(1-S_{H}^{*}\right)-b \beta_{H} \frac{A / \mu_{H}}{N_{H}} S_{H}^{*} I_{V}^{*}, \\
\frac{d}{d t} I_{H}^{*} & =b \beta_{H} \frac{A / \mu_{H}}{N_{H}} S_{H}^{*} I_{V}^{*}-\left(\mu_{H}+\gamma_{H}\right) I_{H}^{*}, \\
\frac{d}{d t} I_{V}^{*} & =b \beta_{V}\left(1-I_{V}^{*}\right) I_{H}^{*}-\mu_{V} I_{V}^{*} .
\end{aligned}
$$

These three equations above describe the changes in time of the variables $S_{H}^{*}, I_{H}^{*}$ and $I_{V}^{*}$, which respectively are the ratios of susceptible humans, infected humans and infected mosquitoes. The system is basically a reduced form of the SIR model of McKermack and Kendrick used together for humans and mosquitoes [12]. The descriptions and the values of the parameters in system (2.1) are given as in Table 1.

Table 1. Values of the parameters of equation system (2.1)

\begin{tabular}{|l|l|l|}
\hline Parameter & Description & Value \\
\hline$\mu_{H}$ & Host birth/death rate & $3.97 \times 10^{-5}$ \\
\hline$\mu_{V}$ & Vector death rate & $7.1429 \times 10^{-2}$ \\
\hline$\gamma_{H}$ & Host recovery rate & $1.4286 \times 10^{-1}$ \\
\hline$b$ & Vector bite rate & Variable \\
\hline$N_{H}$ & Total host population & 50000 \\
\hline$A$ & Vector recruitment & 5000 \\
\hline$\beta_{H}$ & Transmission probability from vector to host & 0.75 \\
\hline$\beta_{V}$ & Transmission probability from host to vector & 1 \\
\hline
\end{tabular}

Various mosquito bite rates have been used in the referred studies. In this study $b=0.25$ has been used whereas the values $0.25,0.45,0.50,0.68$ were the alternatives. The initial values necessary for the calculations are given as:

$$
S_{H}^{*}(0)=0.9999, I_{H}^{*}(0)=1 \times 10^{-4}, I_{V}^{*}(0)=5.71432 \times 10^{-5} .
$$


The values of the parameters and the initial values were taken from the referred studies containing the deterministic model $[8,20]$.

\section{Random Model of Dengue Fever Disease}

The random model of Dengue Fever disease will be built by adding random effect terms to the coefficients of the differential equations in equation system (2.1) to obtain a system of random differential equations. The random effect terms that will be added to the system of differential equations are random variables with Laplace distribution. Every random variable is multiplied with a specific coefficient and added to the parameters for adjusting them to the numerical values of the parameters. The parameters of the equation system become random which in turn transforms the system into a system of random differential equations. A random variable $X$ with Laplace distribution and parameters $(\mu, \sigma)$ has a probability density function with the form

$$
f_{X}(x)=\frac{1}{2 \sigma} \exp \left(-\frac{|x-\mu|}{\sigma}\right), x \in \mathbb{R}, \mu \in \mathbb{R}, \sigma>0 .
$$

A random variable with Laplace distribution and parameters $(0, \sigma)$ can be simulated in MATLAB as the difference of two independent random variables with exponential distribution with parameter $(1 / \sigma)$. Thus, MATLAB is used to simulate random variables $\eta_{i}, i=\overline{(1,5)}$ with Laplace distribution and parameter $(0, \sigma)$.

New parameters will be defined:

$$
\begin{aligned}
& b^{*}=b+c_{1} \eta_{1}, \\
& \mu_{V}^{*}=\mu_{V}+c_{2} \eta_{2}, \\
& \mu_{H}^{*}=\mu_{H}+c_{3} \eta_{3}, \\
& \gamma_{H}^{*}=\gamma_{H}+c_{4} \eta_{4}, \\
& \beta_{H}^{*}=\beta_{H}+c_{5} \eta_{5} .
\end{aligned}
$$

The equation system (2.1) will become random by using these parameters:

$$
\begin{aligned}
\frac{d}{d t} S_{H}^{*} & =\left(\mu_{H}+c_{3} \eta_{3}\right)\left(1-S_{H}^{*}\right)-\left(b+c_{1} \eta_{1}\right)\left(\beta_{H}+c_{5} \eta_{5}\right) \frac{A /\left(\mu_{H}+c_{3} \eta_{3}\right)}{N_{H}} S_{H}^{*} I_{V}^{*}, \\
\frac{d}{d t} I_{H}^{*} & =\left(b+c_{1} \eta_{1}\right)\left(\beta_{H}+c_{5} \eta_{5}\right) \frac{A /\left(\mu_{H}+c_{3} \eta_{3}\right)}{N_{H}} S_{H}^{*} I_{V}^{*}-\left(\mu_{H}+c_{3} \eta_{3}+\gamma_{H}+c_{4} \eta_{4}\right) I_{H}^{*}, \\
\frac{d}{d t} I_{V}^{*} & =\left(b+c_{1} \eta_{1}\right) \beta_{V}\left(1-I_{V}^{*}\right) I_{H}^{*}-\left(\mu_{V}+c_{2} \eta_{2}\right) I_{V}^{*} .
\end{aligned}
$$

The behavior of Dengue Fever disease under random effects with Laplacian distribution will be examined and the differences between the random and deterministic results will be observed by using the new equation system (3.1).

The values of the random quantities obtained from the random variables $\eta_{i}, i=\overline{(1,5)}$ will probably be similar since these random variables are independent and identically distributed. However, it can be seen in the example of $\beta_{H}=0.75$ and $\mu_{H}=3.97 \times 10^{-5}$ that the values of the parameters which the random quantities will be added to can be very different. Thus, the coefficients $c_{i}, i=\overline{(1,5)}$ are determined according to the values of the parameters $b, \mu_{V}, \mu_{H}, \gamma_{H}, \beta_{H}$. In this study, the values of the coefficients $c_{i}, i=\overline{(1,5)}$ are selected to be $5 \%$ of the parameter value to model small randomness in parameters. The selection of the values is also related to the scale parameter of Laplace distribution. Hence, the following values are appropriate:

$$
\begin{aligned}
& c_{1}=0.05 \times 0.25=0.0125, \\
& c_{2}=0.05 \times 7.1429 \times 10^{-2}=3.57145 \times 10^{-3}, \\
& c_{3}=0.05 \times 3.97 \times 10^{-5}=1.985 \times 10^{-6}, \\
& c_{4}=0.05 \times 1.4286 \times 10^{-1}=7.143 \times 10^{-3}, \\
& c_{5}=0.05 \times 0.75=0.0375 .
\end{aligned}
$$


While some of the parameters in the equation system (2.1) are made random to obtain the random model (3.1), some of the parameters are not added random terms. The parameters which do not become random are $N_{H}, A, \beta_{V}$. Here, the parameters $N_{H}$ and $A$ represent total human population and mosquito recruitment rate, respectively, which are assumed to be constant quantities in the model. Thus, random effects were not added to these parameters. The parameter $\beta_{V}$ which represents the transmission probability of disease from humans to mosquitoes has a value of 1 meaning it is a 'sure event'. Therefore, this parameter was not added random effects as well [17]. It should also be noted that the derivation in the random system is treated as a deterministic derivation with varying coefficients in the equations for every different trial of the event.

\section{Numerical Characteristics of the Random Model}

In this part, numerical solutions of the mathematical model of Dengue Fever disease consisting of nonlinear differential equations under random effects are simulated and the results are used to interpret the behavior of the disease under random conditions. Note that the captions of the graphs are only for guidance and the curves describe the changes of the associated starred variables.

\section{Solution Curves}

The solution curves of $S_{H}^{*}, I_{H}^{*}$ and $I_{V}^{*}$ are obtained for the equation system (3.1) as below (Figure 1).
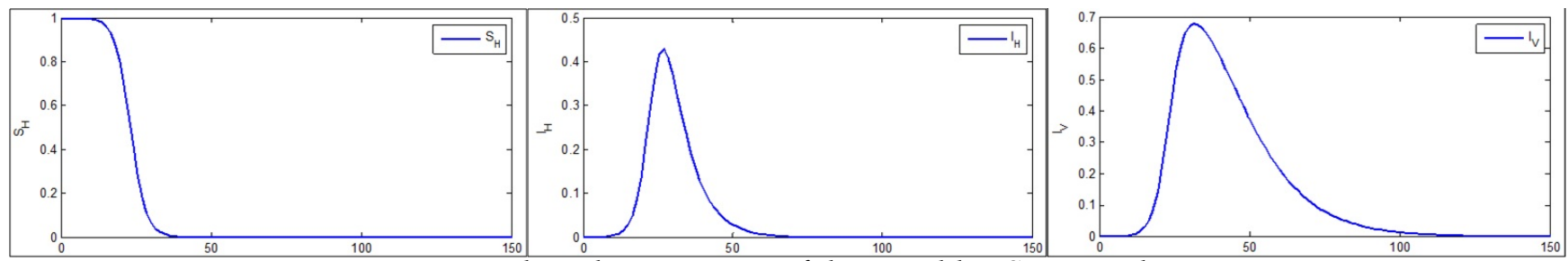

Figure 1. The solution curves of the variables $S_{H}^{*}, I_{H}^{*}$ and $I_{V}^{*}$

As it can be seen in the figures, the ratios of the susceptible humans, infected humans and the infected mosquitoes go down to zero in time. This implies the eradication of the disease. The maximum and minimum values for the variables $S_{H}^{*}, I_{H}^{*}$ and $I_{V}^{*}$ are obtained in the results as below (Table 2):

Table 2. Maximum and minimum values of the solution curves

\begin{tabular}{|l|l|l|l|}
\hline & $S_{H}^{*}$ & $I_{H}^{*}$ & $I_{V}^{*}$ \\
\hline Maximum Value & 0.9999 & 0.4288 & 0.6787 \\
\hline Time of Maximum & 0 & 27 & 31.5 \\
\hline Minimum Value & 0.0003003 & $0.8408 \times 10^{-6}$ & $5.714 \times 10^{-5}$ \\
\hline Time of Minimum & 55.5 & 150 & 0 \\
\hline
\end{tabular}

The graph below shows the solution curves of $S_{H}^{*}, I_{H}^{*}$ and $I_{V}^{*}$ together for an overall analysis of the course and the random behavior of the disease (Figure 2).

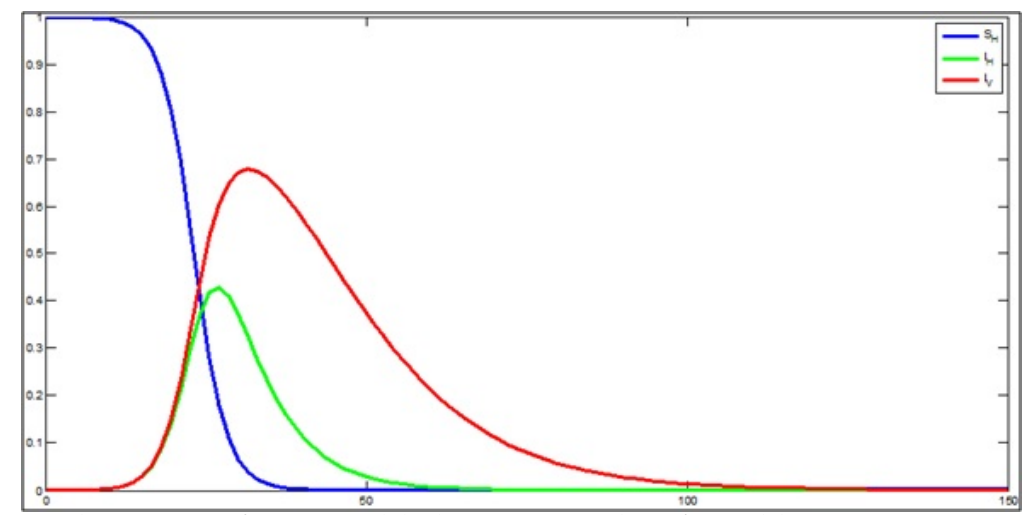

Figure 2. The ratios of susceptible humans with infected humans \& mosquitoes 
The following comments can be made on the disease using these graphs and extremum values. About $9-10$ days after the start of the disease, the number of susceptible humans decreases rapidly and the numbers of infected humans and mosquitoes increase rapidly. Considering the fact that the human and mosquito populations are constant, this indicates a spread of the disease in the population. A short time after the infected humans reach their maximum value (0.4288) at $t=27$ and start decreasing, infected mosquitoes hit their maximum value $(0.6787)$ at $t=31.5$ and then start decreasing. This could indicate that the decrease in infected humans affects infected mosquitoes and causes a decrease in infected mosquitoes as well. After this stage, the number of infected humans and mosquitoes decrease rapidly and the number of susceptible humans becomes almost zero. This indicates that the recovered fraction of the human population starts growing and the disease starts to become eradicated from the population. Hence, it can be concluded that the disease spreads to a certain fraction of the human population and after that stage slowly decreases in the human and mosquito population only to leave the environment after about 100 days. The characteristics of the random system are given below by using $10^{5}$ simulations created in MATLAB.

\section{Expected Values}

The expected values of $S_{H}^{*}, I_{H}^{*}$ and $I_{V}^{*}$ are obtained for the equation system (3.1) as below (Figure 3).

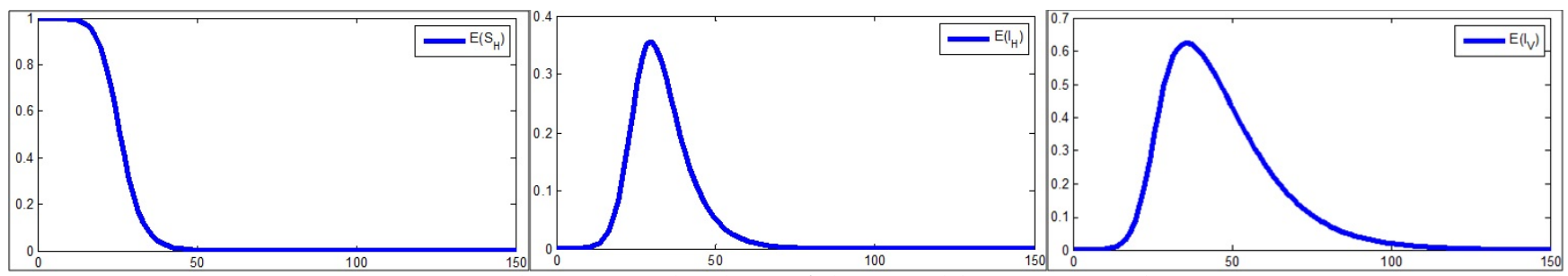

Figure 3. The expected values of the variables $S_{H}^{*}, I_{H}^{*}$ and $I_{V}^{*}$

As seen in the graphs above, the expected values and the solution curves of the susceptible humans, infected humans and infected mosquitoes are very similar. Similarity between the expected values and the solution curves of random variables is expected. The maximum and minimum values for the variables $E\left(S_{H}^{*}\right), E\left(I_{H}^{*}\right)$ and $E\left(I_{V}^{*}\right)$ are obtained in the results as below (Table 3):

Table 3. Minimum and maximum values in the expected values

\begin{tabular}{|l|l|l|l|}
\hline & $E\left(S_{H}^{*}\right)$ & $E\left(I_{H}^{*}\right)$ & $E\left(I_{V}^{*}\right)$ \\
\hline Maximum Value & 0.9999 & 0.3568 & 0.6264 \\
\hline Time of Maximum & 0 & 30 & 36 \\
\hline Minimum Vale & 0.0006801 & $1.157 \times 10^{-5}$ & $5.714 \times 10^{-5}$ \\
\hline Time of Minimum & 73.5 & 150 & 0 \\
\hline
\end{tabular}

Although there is a similarity between the figures of the expected values and the solution curves of the variables $S_{H}^{*}, I_{H}^{*}$ and $I_{V}^{*}$, it can be seen from the extremum values table that there are numerical differences between the expectations and the solutions. It can be said that the 'expected value' characteristic of this model successfully models the random behavior of the disease but errors occur in estimating the numerical data. Increasing the number of simulations could decrease the differences between the expectations and the solutions and thus increase the accuracy of the numerical characteristics.

\section{Variances}

The variances of $S_{H}^{*}, I_{H}^{*}$ and $I_{V}^{*}$ are obtained for the equation system (3.1) as below (Figure 4).
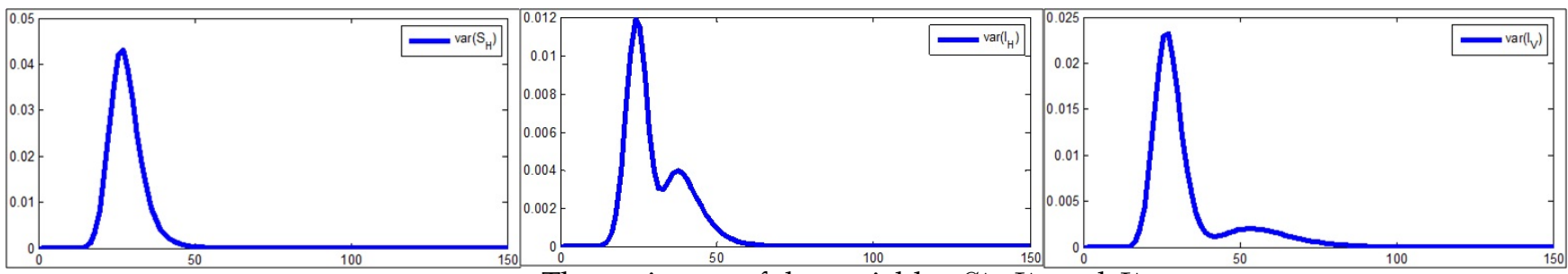

Figure 4. The variances of the variables $S_{H}^{*}, I_{H}^{*}$ and $I_{V}^{*}$ 
It can be seen in the graphs that the variances of the ratios of the susceptible humans, infected humans and infected mosquitoes increase and decrease in similar time periods of the process. Although there is a small jump in the variances of infected humans and mosquitoes after they start decreasing, all of the variances decrease to zero after a certain time. The maximum and minimum values for the variables $\operatorname{Var}\left(S_{H}^{*}\right), \operatorname{Var}\left(I_{H}^{*}\right)$ and $\operatorname{Var}\left(I_{V}^{*}\right)$ are obtained in the results as below (Table 4).

Table 4. Maximum and minimum values in the variances

\begin{tabular}{|l|l|l|l|}
\hline & $\operatorname{Var}\left(S_{H}^{*}\right)$ & $\operatorname{Var}\left(I_{H}^{*}\right)$ & $\operatorname{Var}\left(I_{V}^{*}\right)$ \\
\hline Maximum Value & 0.04304 & 0.01191 & 0.02326 \\
\hline Time of Maximum & 27 & 24 & 27 \\
\hline Minimum Vale & $8.931 \times 10^{-25}$ & $9.936 \times 10^{-33}$ & $1.432 \times 10^{-33}$ \\
\hline Time of Minimum & 0 & 0 & 0 \\
\hline
\end{tabular}

Considering the solution curves of the variables, it can be said that the similarity in the variances of $S_{H}^{*}, I_{H}^{*}$ and $I_{V}^{*}$ indicates an increase in the variance for the time period in which the disease starts to leave the population, especially between $t=15$ and $t=50$. A high variance in the time period where the disease grows to a maximum level and starts decreasing indicates that there is a high deviation between the expectations and the realizations of the event. Although a high variance in this unstable phase of the disease might be expected, more successful estimations of the expected values could cause a decrease in these values.

\section{Standard Deviations}

The standard deviations of $S_{H}^{*}, I_{H}^{*}$ and $I_{V}^{*}$ are obtained for the equation system (3.1) as below (Figure 5).
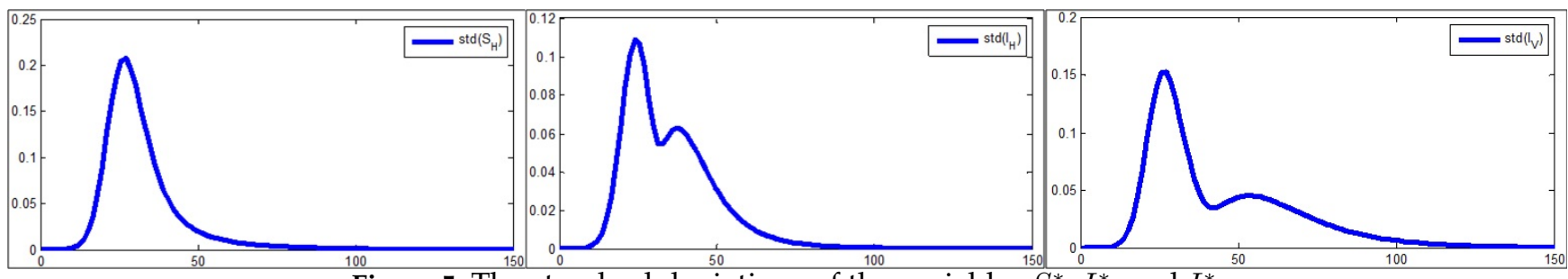

Figure 5. The standard deviations of the variables $S_{H}^{*}, I_{H}^{*}$ and $I_{V}^{*}$

The graphs show that the standard deviations of the ratios of the susceptible humans, infected humans and infected mosquitoes increase and decrease in similar periods to the variances. Another similarity is the slight jump in the graphs of the infected humans and mosquitoes just after they start decreasing. Again, all deviations go down to zero after a certain period. These similarities are expected since standard deviation is defined as the square root of variance. The maximum and minimum values for the variables $\operatorname{std}\left(S_{H}^{*}\right), \operatorname{std}\left(I_{H}^{*}\right)$ and $\operatorname{std}\left(I_{V}^{*}\right)$ are obtained in the results as (Table 5).

Table 5. Maximum and minimum values in the standard deviations

\begin{tabular}{|l|l|l|l|}
\hline & $s t d\left(S_{H}^{*}\right)$ & $s t d\left(I_{H}^{*}\right)$ & $s t d\left(I_{V}^{*}\right)$ \\
\hline Maximum Value & 0.2075 & 0.1092 & 0.1525 \\
\hline Time of Maximum & 27 & 24 & 27 \\
\hline Minimum Vale & $9.45 \times 10^{-13}$ & $9.968 \times 10^{-17}$ & $3.7852 \times 10^{-17}$ \\
\hline Time of Minimum & 0 & 0 & 0 \\
\hline
\end{tabular}

Similar comments to the can be made for the changes in standard deviations as well. Considering the table of extremum values above, it can be said that there are notable deviations from the expectations in some cases. 


\section{Confidence Intervals}

The confidence intervals of $S_{H}^{*}, I_{H}^{*}$ and $I_{V}^{*}$ are obtained for the equation system (3.1) as below (Figure 6). Confidence intervals are defined by

$$
\begin{aligned}
& {\left[E\left(S_{H}^{*}\right)-K \cdot \operatorname{std}\left(S_{H}^{*}\right), E\left(S_{H}^{*}\right)+K \cdot \operatorname{std}\left(S_{H}^{*}\right)\right],} \\
& {\left[E\left(I_{H}^{*}\right)-K \cdot \operatorname{std}\left(I_{H}^{*}\right), E\left(I_{H}^{*}\right)+K \cdot \operatorname{std}\left(I_{H}^{*}\right)\right],} \\
& {\left[E\left(I_{V}^{*}\right)-K \cdot \operatorname{std}\left(I_{V}^{*}\right), E\left(I_{V}^{*}\right)+K \cdot \operatorname{std}\left(I_{V}^{*}\right)\right] .}
\end{aligned}
$$
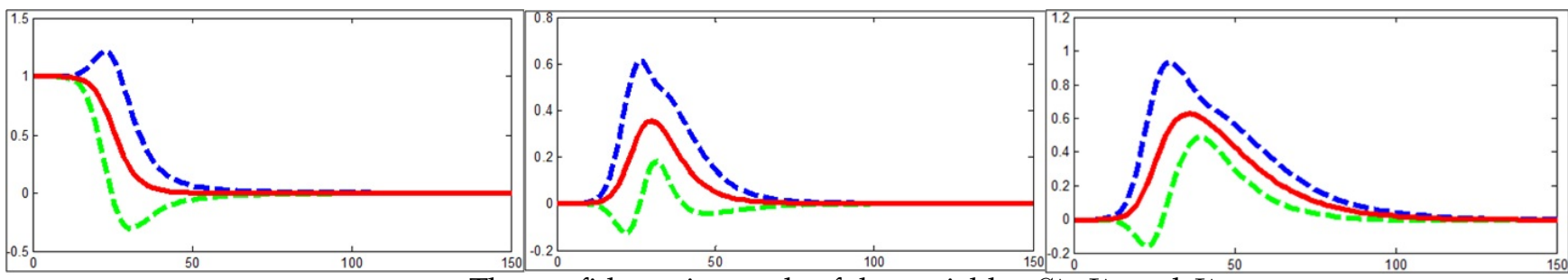

Figure 6. The confidence intervals of the variables $S_{H}^{*}, I_{H}^{*}$ and $I_{V}^{*}$

The graphs for the confidence intervals of the expectations of the ratios of the susceptible humans, infected humans and infected mosquitoes are in accordance with the changes in the expected values and variance-deviations of the same variables (blue-dashed lines represent upper limit, green-dashed lines represent lower limit). Extremum values are seen below (Table 6).

Table 6. Maximum and minimum values in the confidence intervals

\begin{tabular}{|l|l|l|l|}
\hline & $E\left(S_{H}^{*}\right) \mp K \cdot \operatorname{std}\left(S_{H}^{*}\right)$ & $E\left(I_{H}^{*}\right) \mp K \cdot \operatorname{std}\left(I_{H}^{*}\right)$ & $E\left(I_{V}^{*}\right) \mp K \cdot \operatorname{std}\left(I_{V}^{*}\right)$ \\
\hline Maximum Value & 1.212 & 0.6132 & 0.9372 \\
\hline Time of Maximum & 22.5 & 27 & 30 \\
\hline Minimum Value & -0.3025 & -0.1248 & -0.1583 \\
\hline Time of Minimum & 30 & 21 & 22.5 \\
\hline
\end{tabular}

$K=3$ gives approximately $99 \%$ confidence intervals for the expected values of the variables $S_{H}^{*}, I_{H}^{*}$ and $I_{V}^{*}$. $K=2$ could also be used to obtain an approximately $95 \%$ interval. The most unstable period of the disease (between $t=15$ and $t=50$ ) causes deviations between the real values and the expected values, which in turn affects the standard deviations. It can be seen in the graphs that the confidence intervals grow larger in the period where there are increases in the standard deviations.

\section{Third Central Moments}

The third central moments of $S_{H}^{*}, I_{H}^{*}$ and $I_{V}^{*}$ are obtained for the equation system (3.1) as below (Figure 7).
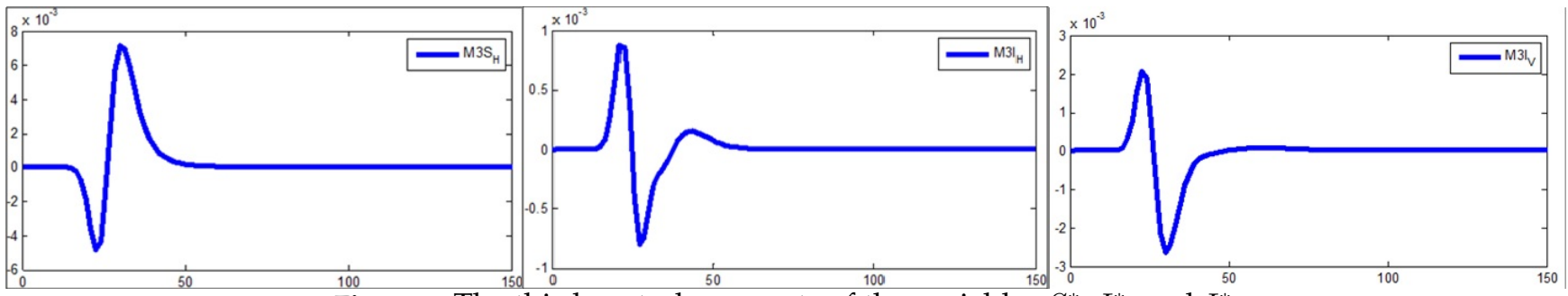

Figure 7. The third central moments of the variables $S_{H}^{*}, I_{H}^{*}$ and $I_{V}^{*}$

Extremum values of the third central moments of the variables are given below (Table 7).

\section{Fourth Central Moments}

The fourth central moments of $S_{H}^{*}, I_{H}^{*}$ and $I_{V}^{*}$ are obtained for the equation system (3.1) as below (Figure 8).

Extremum values of the fourth central moments of the variables are given below (Table 8). 
Table 7. Maximum and minimum values in the third central moments

\begin{tabular}{|l|l|l|l|}
\hline & $E\left(\left(S_{H}^{*}-E\left(S_{H}^{*}\right)\right)^{3}\right)$ & $E\left(\left(I_{H}^{*}-E\left(I_{H}^{*}\right)\right)^{3}\right)$ & $E\left(\left(I_{V}^{*}-E\left(I_{V}^{*}\right)\right)^{3}\right)$ \\
\hline Maximum Value & 0.007172 & 0.008774 & 0.002073 \\
\hline Time of Maximum & 30 & 21 & 22.5 \\
\hline Minimum Value & -0.004844 & -0.008039 & -0.002634 \\
\hline Time of Minimum & 22.5 & 27 & 30 \\
\hline
\end{tabular}
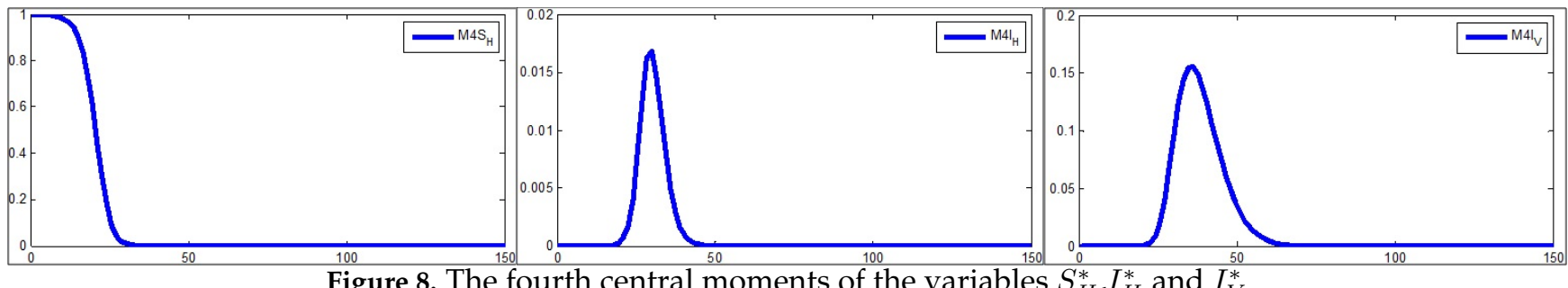

Figure 8. The fourth central moments of the variables $S_{H}^{*}, I_{H}^{*}$ and $I_{V}^{*}$

Table 8. Maximum and minimum values in the fourth central moments

\begin{tabular}{|l|l|l|l|}
\hline & $E\left(\left(S_{H}^{*}-E\left(S_{H}^{*}\right)\right)^{4}\right)$ & $E\left(\left(I_{H}^{*}-E\left(I_{H}^{*}\right)\right)^{4}\right)$ & $E\left(\left(I_{V}^{*}-E\left(I_{V}^{*}\right)\right)^{4}\right)$ \\
\hline Maximum Value & 0.9996 & 0.01683 & 0.1557 \\
\hline Time of Maximum & 0 & 30 & 36 \\
\hline Minimum Value & $2.893 \times 10^{-10}$ & $1 \times 10^{-16}$ & $1.066 \times 10^{-17}$ \\
\hline Time of Minimum & 150 & 0 & 0 \\
\hline
\end{tabular}

\section{Skewness}

The skewness coefficients of $S_{H}^{*}, I_{H}^{*}$ and $I_{V}^{*}$ are obtained for the equation system (3.1) as below (Figure 9).
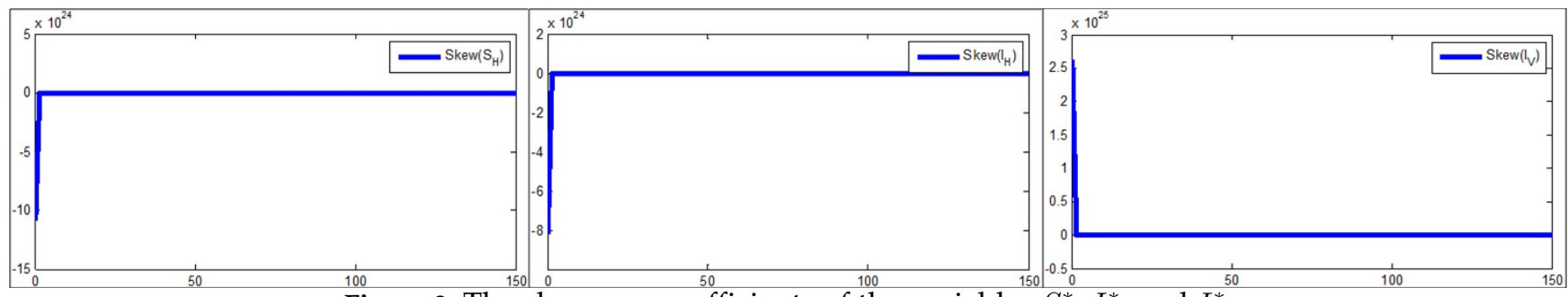

Figure 9. The skewness coefficients of the variables $S_{H}^{*}, I_{H}^{*}$ and $I_{V}^{*}$

Extremum values of the skewness coefficients of the variables are given below (Table 9).

Table 9. Maximum and minimum values in the skewness coefficients

\begin{tabular}{|l|l|l|l|}
\hline & $\operatorname{Skew}\left(S_{H}^{*}\right)$ & $\operatorname{Skew}\left(I_{H}^{*}\right)$ & $\operatorname{Skew}\left(I_{V}^{*}\right)$ \\
\hline Maximum Value & 215.6 & 263.7 & $2.626 \times 10^{25}$ \\
\hline Time of Maximum & 96 & 144 & 0 \\
\hline Minimum Value & $-1.088 \times 10^{25}$ & $-8.256 \times 10^{24}$ & -5.436 \\
\hline Time of Minimum & 0 & 0 & 36 \\
\hline
\end{tabular}

\section{Kurtosis}

The kurtosis coefficients of $S_{H}^{*}, I_{H}^{*}$ and $I_{V}^{*}$ are obtained for the equation system (3.1) as below (Figure 10).

Extremum values of the kurtosis coefficients of the variables are given below (Table 10).

The graphs of the solution curves, expected values, variances, standard deviations and confidence intervals of the variables of the random model of Dengue Fever disease are given above. The extremum values of the results and the comments on the graphs had also been given along with these results. Mathematical implications of these results and the comparison of the random results with the deterministic results must be given to discuss the contribution of random effects to mathematical modeling. 


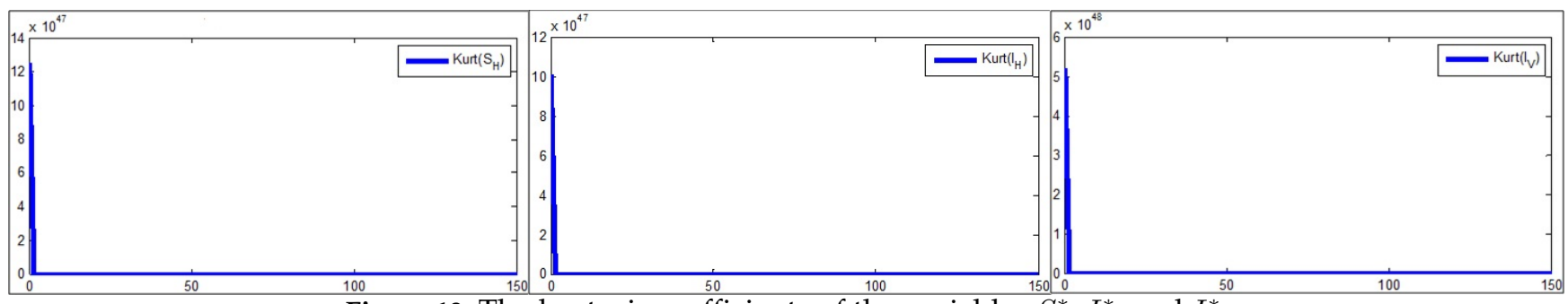

Figure 10. The kurtosis coefficients of the variables $S_{H}^{*}, I_{H}^{*}$ and $I_{V}^{*}$

Table 10. Maximum and minimum values in the skewness coefficients

\begin{tabular}{|l|l|l|l|}
\hline & $\operatorname{Kurt}\left(S_{H}^{*}\right)$ & $\operatorname{Kurt}\left(I_{H}^{*}\right)$ & $\operatorname{Kurt}\left(I_{V}^{*}\right)$ \\
\hline Maximum Value & $1.253 \times 10^{48}$ & $1.013 \times 10^{48}$ & $5.198 \times 10^{48}$ \\
\hline Time of Maximum & 0 & 0 & 0 \\
\hline Minimum Value & 6.184 & 10.22 & 11.83 \\
\hline Time of Minimum & 30 & 21 & 21 \\
\hline
\end{tabular}

\section{Comments of the Disease}

In this study, the deterministic mathematical of model of Dengue Fever disease has been transformed into a system of nonlinear random differential equations by changing its parameters into random variables. Before making any comments on the disease using the graphs of the simulations of the numerical results for the equation system (3.1) given above, two important constraints of the equation system must be remembered:

$$
\begin{aligned}
& N_{H}=S_{H}+I_{H}+R_{H}, \frac{d}{d t} N_{H}=0 \\
& N_{V}=S_{V}+I_{V}, N_{V} \rightarrow \frac{A}{\mu_{H}}
\end{aligned}
$$

Note that the variables describe the amounts of the model components they define while the starred variables stand for the ratios of the same components in the population. The variables in the graphs are $S_{H}^{*}, I_{H}^{*}$ and $I_{V}^{*}$, i.e. the ratios of susceptible humans, infected humans and infected mosquitoes, and by using the condition $N_{H}=S_{H}+I_{H}+R_{H}$, we can obtain a new equation for these variables as

$$
S_{H}^{*}+I_{H}^{*}+R_{H}^{*}=1
$$

which would let us make comments for the variable $R_{H}^{*}$ (ratio of recovered humans) using the results for $S_{H}^{*}, I_{H}^{*}$. Similarly, comments can be made for $S_{V}^{*}$ using the result for $I_{V}^{*}[8]$.

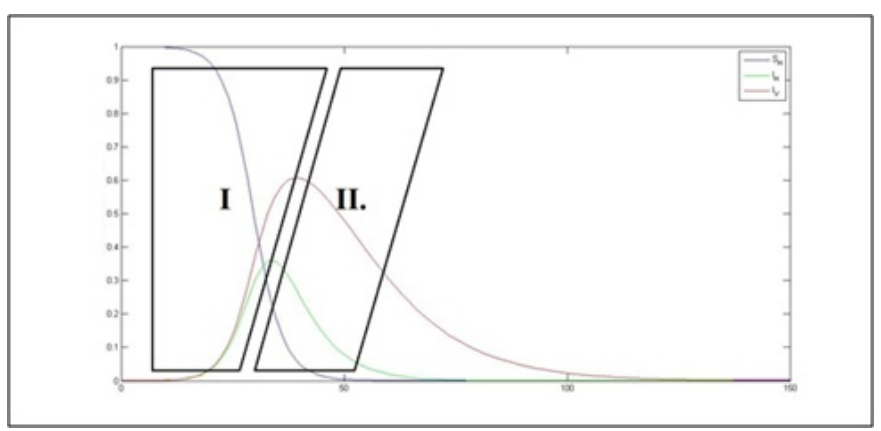

Figure 11. Analysis stages of solution curves

Stage I. Considering there is only a little change in the first parts of the graphs of the variables $S_{H}^{*}, I_{H}^{*}$ and $I_{V}^{*}$, it can be said that the progress of the disease is very slow at this stage. The disease keeps a slow progress until $t=10$ after which the progress accelerates. The increase in the numbers of infected humans and mosquitoes shows an exponential behavior, hence the small initial value $\left(I_{V}^{*}(0)=5.71432 \times 10^{-5}\right)$ of the Dengue Fever virus could be thought of as the reason behind the slow progress in the initial stages of the disease which rapidly increases after 
this stage. As the virus spreads in the population, the disease progresses exponentially.

Stage II. The second stage of the examination of graphs is the decline of the disease. When the curves of the variables $I_{H}^{*}$ and $I_{V}^{*}$ are examined, it can be seen that firstly $I_{H}^{*}$, the ratio of the infected humans, reaches its maximum and starts decreasing. Shortly after this point, $I_{V}^{*}$, the ratio of infected mosquitoes, reaches its maximum and starts decreasing. Considering the flowchart of the disease and the equation system (3.1), it is clear that the variables $I_{V}^{*}$ and $I_{H}^{*}$ are dependent, hence it can be concluded that the decline in the ratio of infected humans causes a decline in the ratio of infected mosquitoes. The ratios $S_{H}^{*}$ and $I_{H}^{*}$ converge to zero after this stage, hence the ratio $R_{H}^{*}$ converges to 1 meaning that almost all of the population becomes recovered. Similarly, the convergence of the ratio $I_{V}^{*}$ to zero indicates that the disease has been removed from the mosquito population as well.

\section{Comparison of the Deterministic and the Random Models}

After examining the course of the disease and the virus dynamics under random conditions, the results of the study titled "Mathematical Study of Biting Rates of Mosquitoes in Transmission of Dengue Disease" by Phaijoo and Gurung in 2015 can be examined to analyze the differences between the deterministic and the random cases. The following graph has been obtained from the analysis of the deterministic model, which was used to build the random model [20]:

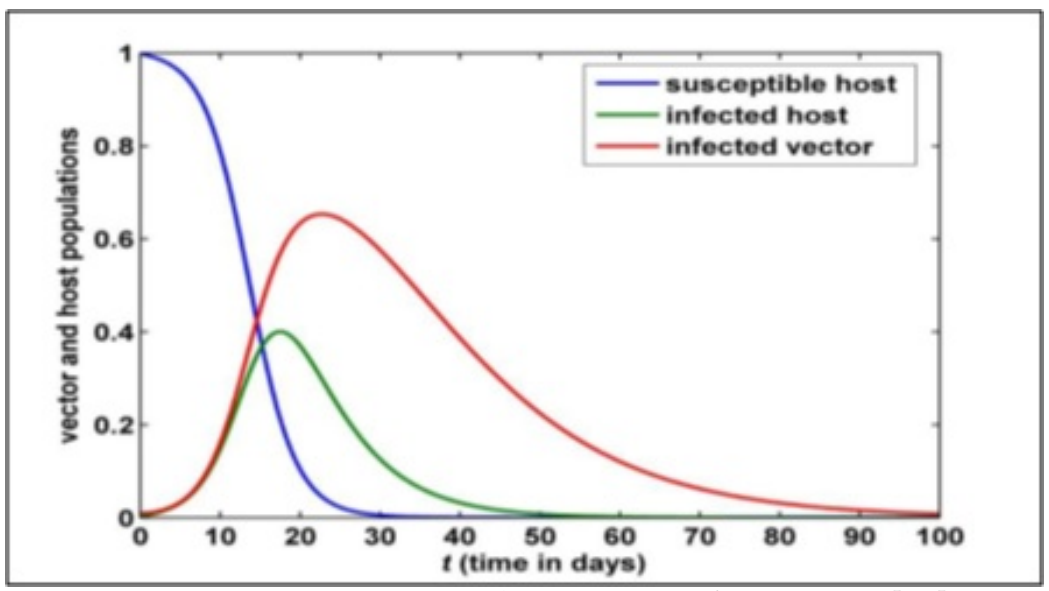

Figure 12. Deterministic solution curves for $b=0.45$ [20]

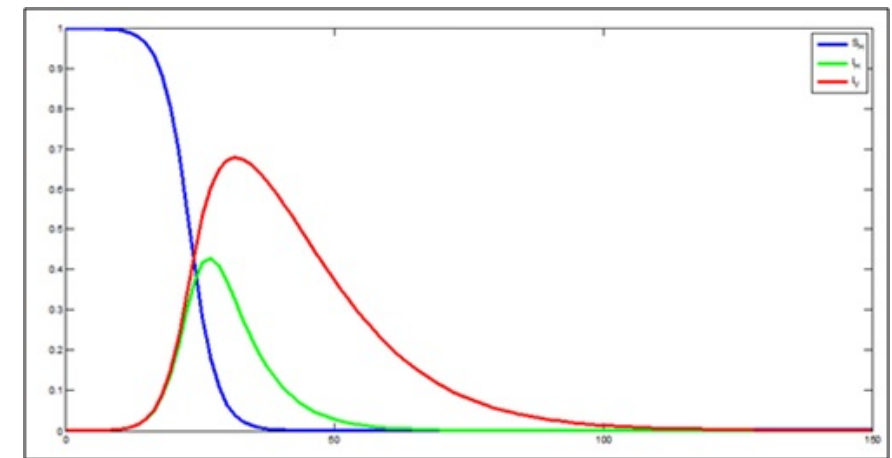

Figure 13. Random results of the analysis with the same parameter values

It should be noted that the variables $S_{H}^{*}, I_{H}^{*}$ and $I_{V}^{*}$ show very similar behavior in both deterministic and random cases. This is a proof of the fact that the method used for the random analysis produces sensible results. The first difference that can be noticed between the two graphs is that the disease begins its spread a short while after the beginning of the analysis in the random case, whereas this increase starts right at the beginning in the deterministic case. The slow progress until $t=10$ in the random case can not be seen in the deterministic case. This almost-contant period is also the reason behind the larger time interval used for the random model. This stable phase in the random case also affects the timing of the maximum points of the random variables, enabling the deterministic extremum points to be reached earlier. 
Another difference that should be noted between the deterministic and the random cases is the maximum values of the infected humans and mosquitoes. The variable $I_{V}^{*}$ barely exceeds the 0.6 limit in the deterministic case whereas it almost reaches 0.7 in the random case, while the variable $I_{H}^{*}$ exceeds the value 0.4 in the random case but not in the deterministic case. Two of the most obvious differences mentioned between the random and the deterministic cases can lead to potentially different approaches in battling this disease. For instance, the total eradication of the disease lasts about 50 days in the deterministic case, whereas it lasts about 75 days in the random case. Similarly in the random case, the disease spreads to $40 \%$ of the population in the worst scenario, whereas this value is less in the deterministic case.

Mathematical models are expected to guide the efforts to battle diseases and find treatments as well as to show the way to health policies. Deterministic models that neglect "small" variations in the values of the parameters which occur differently in different parts of the world could cause serious misleading which is obviously pointed out in the two examples above. Thus, the need for a random study and the advantages of random analysis in modeling of diseases has been seen with the example of Dengue Disease.

\section{Conclusion}

Random modeling of diseases offers advantages in terms of the opportunities for additional analyses and the probability of producing more accurate results for real life cases. A similar approach to the study of Phaijoo and Gurung in 2015 can be followed to analyze various values of the parameters and the effects of the changes in these parameters on the disease. This could produce results which could guide treatment studies by showing the impacts of parameters in the results.

Another type of random modeling, stochastic modeling, could be used by using stochastic differential equations, to produce a more active contribution to modeling studies through the use of the tools of stochastic calculus. While random modeling studies require less effort for producing valuable information on the disease, stochastic calculus promises more depth in the results.

Considering the results for the expectations and the variances, increasing the number of simulation can be thought of as a method for increasing the accuracy of these results. Using a greater number of simulations can produce values for the characteristics which would be closer to the real values.

Calculation of equilibrium points and the stability of these points could provide a different perspective and useful results in random modeling and stochastic analysis.

Finally, a study on the distribution used for the random effects could be made. Various distributions could be used on different parameters spontaneously or separately to compare their results, which would enrich random modeling studies of infectious diseases.

\section{References}

[1] Bailey, N., The Mathematical Theory of Infectious Diseases and its Applications, Griffin, London, 1975.

[2] Bhatt, S., Gething, P.W., Brady, O.J., Messina, J.P., Farlow, A.W., Moyes, C.L. et al., The global distribution and burden of dengue. Nature, 496 (2013), 504-507.

[3] Brady, O.J., Gething, P.W., Bhatt, S., Messina, J.P., Brownstein, J.S., Hoen, A.G. et al., Refining the global spatial limits of dengue virus transmission by evidence-based consensus. PLOS Negl Trop Dis 6 (20120), no. 8.

[4] Bronson, R., Schaum's Outline of Differential Equations, 4th Edition, McGraw-Hill Education, New York, 2014.

[5] Butcher, J.C., Numerical Methods for Ordinary Differential Equations, John Wiley \& Sons, New York, 2008.

[6] Cyganowski, S., Kloeden, P. and Ombach, J., From Elementary Probability to Stochastic Differential Equations with MAPLE®, Springer-Verlag, New York, 2001.

[7] Dietz, K., Transmission and control of arbovirus diseases. In D. Ludwig and K. L. Cooke, editors, Epidemiology, 104-121. SIAM, 1975. 
[8] Esteva, L. and Vargas, C., Analysis of a dengue disease transmission model. Mathematical Biosciences, 150 (1998), 131-151.

[9] Feller W., An Introduction to Probability Theory and Its Applications, Volume I, 3rd Edition John Wiley \& Sons, Inc., New York, 1968.

[10] Feller W., An Introduction to Probability Theory and Its Application, Volume II, John Wiley \& Sons, Inc., New York, 1971.

[11] Imran, M., Hassan, M., Dur-E-Ahmad, M. and Khan, A., A comparison of a deterministic and stochastic model for Hepatitis C with an isolation stage. Journal of Biological Dynamics, 7 (2013), no. 1, 276-301.

[12] Kermack, W.O. and McKendrick, A.G., A Contribution the Mathematical Theory of Epidemics. Proceedings of The Royal Society A, 115 (1927), no. 772, 700-721.

[13] Kloeden, P.E. and Platen, E., Numerical Solution of Stochastic Differential Equations, Second Edition, SpringerVerlag, New York, 1995.

[14] Kolmogorov A. N., Foundations of the Theory of Probability, Chelsea Publishing Company, New York, 1956.

[15] Lahrouz, A., Omari, L., Kiouach, D. and Belmaati, A., Deterministic and Stochastic Stability of a Mathematical Model of Smoking. Statistics and Probability Letters, 81 (2011), 1276-1284.

[16] Martcheva, M., An Introduction to Mathematical Epidemiology, Springer Science+Business Media, New York, 2015.

[17] Merdan M., Bekiryazici Z. and Kesemen T., Stochastic and Deterministic Stability of Models for Hepatitis C, 7th International Conference on Mathematical Analysis, Differential Equations and their Applications, September 2015, Baku, Abstracts Book, p. 114.

[18] Merdan, M., and Khaniyev, T., On the Behaviour of Solutions under the Influence of Stochastic Effect of Avian-Human Influenza Epidemic Model. International Journal of Biotechnology and Biochemistry, 4 (2008), no. 1, 75-100.

[19] Pang, L., Zhao, Z., Liu, S., Zhang, X., A Mathematical Model Approach for Tobacco Control in China. Applied Mathematics and Computation, 259 (2015), 497-509.

[20] Phaijoo, G.R. and Gurung, D.B., Mathematical Study of Biting Rates of Mosquitoes in Transmission of Dengue Disease, Journal of Science. Engineering and Technology, 11 (2015), no. 2, 25-33.

[21] Shiryayev A. N., Gradute Texts in Mathematics: Probability, Spring Science+ Business Media, LLC, New York, 1984.

[22] Soong, T.T., Random Differential Equations in Science and Engineering, Academic Press Inc., New York, 1973.

[23] Tan, W. and Wu, H., Stochastic Modeling of the Dynamics of CD4+ T-Cell Infection by HIV ad Some Monte Carlo Studies. Mathematical Biosciences, 147 (1998), 173-205.

[24] Yaacob, Y., Analysis of a Dengue Disease Transmission Model without Immunity. Matematika, 23 (2007), no. 2, 75-81.

[25] World Health Org., Dengue and severe dengue, http://www.who.int/mediacentre/factsheets/fs117/en/, 23 March 2016. 


\section{Affiliations}

ZAFER BEKIRYAZICI

AdDRESS: Recep Tayyip Erdogan University, Dept. of Mathematics, 53100, Rize-Turkey.

E-MAIL: zafer.bekiryazici@erdogan.edu.tr

MEHMET MERDAN

ADDRESS: Gumushane University, Dept. of Mathematical Engineering, 29100, Gumushane-Turkey.

E-MAIL: mmerdan@gumushane.edu.tr

TUlAy KESEMEN

ADDRESS: Karadeniz Technical University, Dept. of Mathematics, 61080, Trabzon-Turkey.

E-MAIL: tkesemen@gmail.com

MOHAMMED NAJMULDEEN

AdDRESs: Karadeniz Technical University, Dept. of Mathematics, 61080, Trabzon-Turkey.

E-MAIL: mmt_terzibasi@yahoo.com 